\title{
COMPUTER SIMULATIONS OF ELASTIC CONSTANTS WITHOUT CALCULATING DERIVATIVES OF THE INTERACTION POTENTIAL
}

\author{
K. W. WO J C I E C H O W S K I \\ Institute of Molecular Physics, Polish Academy of Sciences, \\ Smoluchowskiego 17/19, 60-179 Poznań, Poland \\ email:kww@man.poznan.pl
}

\begin{abstract}
Two Monte Carlo methods of elastic constants determination which do not require using any derivatives of the interaction potential between the particles are described. The first method corresponds to the free energy differentiation with respect to deformation in the fixed box ensemble $(N V T)$. The second method is based on the analysis of the box fluctuations in the constant pressure ensemble with variable box shape $(N p T)$. Its additional advantage is that it does not require any knowledge of the reference state of the system prior to simulations.
\end{abstract}

\section{INTRODUCTION}

The elastic properties of materials are of interest both for applications and for fundamental research [1-4]. Computer simulations offer several methods to determine the elastic properties of models of various systems [5-25], Typically, the simulation methods of the elastic properties determination are more complex and much more time-consuming than the methods used for determination of the equation of state. For these reasons there is still a need for searching for new methods, as well as for modifications of the known methods, which can simplify or speed up calculations of the elastic properties.

In most of the known methods used for determination of the elastic properties of solids derivatives of the interaction potential are required to compute the microscopic pressure or microscopic elastic constants $[5,7,12,13,15-17,21,21-25]$, These derivatives can be complicated to obtain and to apply when complex molecules are considered or non-analytic potentials are used. In the approaches discussed in the present paper one avoids calculating and averaging derivatives of the interaction potential.

The first of the approaches discussed in this paper is in fact the most natural method which does not require microscopic formulae including the interaction potential derivatives. This method, not yet discussed in the literature, is based on numerical differentiation (with respect to the strain components) of the free energy of a system studied. In this approach, the free energy must be computed for various deformations of the reference sample, and the results of such simulations are used to obtain smooth fits representing the free energy dependence of the deformations. To obtain the free energy of each deformed sample one can use, e.g., 
the Frenkel-Ladd method [26, 27]. The drawback of this method, further referred to as the $N V T$ method, is that one needs to use many runs (each corresponding to a different deformation of the sample) to determine the free energy dependence on the system deformation. Moreover, the simulations must be accurate enough to allow one for double differentiation of the obtained free energy fits with respect to deformation.

In some of the known simulation methods one can establish the complete elastic properties of a studied model within a single run. These methods [5, 6, 8-11, 18-22, 24], based on analysis of fluctuations of either the stress tensor or the stain tensor, are usually referred to as the fluctuation methods [21], When one is interested in obtaining elastic properties of systems under isotropic stress, constant pressure simulations in a periodic box of variable shape are of special interest [20, 28-30]; they are further referred to as $N p T$ simulations. The latter simulations, which constitute the second approach discussed in the present work, have a few advantages with respect to other methods, (i) Firstly, all the elastic constants (compliances) can be computed within a single run. (ii) Secondly, in contrast to other methods, the reference state (which corresponds to the equilibrium state) can be computed within the same run in which the elastic properties are determined from fluctuations of the box edges. (The other methods require knowing the reference state in advance because it is used as an input for explicit computing the strain. Hence, an extra simulation is necessary for low-symmetry models.) (iii) Thirdly, as it occurs in the case of the described above $N V T$ simulations, also in the constant pressure simulations one avoids calculating and averaging derivatives of the interaction potential. Unfortunately, the $N p T$ calculations based on strain fluctuations are known to be rather slowly convergent [9]. This drawback can be overcome, however, if studies of small samples are sufficient to characterize the model systems of interest.

The aim of the present paper is to discuss the theoretical background and to present the basic formulae of both the above approaches. The structure of the paper is as follows. In Sec. 2 we describe the $N V T$ simulation method. In Sec. 3 we present the $N p T$ simulations of the elastic constants. The last section (Sec. 4) contains the summary and conclusions.

\section{NVT SIMULATIONS IN FIXED PERIODIC BOXES}

The (Lagrange) strain tensor [4] which describes deformations of the system can be expressed by the box matrix [8], h, and the reference box matrix, $\mathbf{H}$,

where it has been assumed, for convenience, that the matrices $\mathbf{h}, \mathbf{H}$ are symmetric.

The elastic free energy $\Delta F$ of a system which deformation from the reference state (corresponding to the stress tensor $\mathbf{T}$ and the reference state matrix $\mathbf{H}$ ) is described by the matrix $\mathbf{h}=\mathbf{H}+\boldsymbol{\delta}^{\prime}$ (where all the components of the matrix $\boldsymbol{\delta}^{\prime}$ tend to zero) can be written as [4] 


$$
\Delta F / V_{p}=\sum_{i j} \tau_{i j} \varepsilon_{i j}+\frac{1}{2} \sum_{i j k l} C_{i j k l} \varepsilon_{i j} \varepsilon_{k l}
$$

Differentiating the free energy with respect to the components of the matrix $\boldsymbol{\delta}^{\prime}$ at the reference state one obtains simple relations from which the components of the stress tensor and the tensor of the elastic constants can be determined as functions of the (first and second order) derivatives. This procedure will be illustrated below in the case of a solid of cubic symmetry which is subjected to the isotropic external pressure $\boldsymbol{\tau}=-p \mathbf{I}$, where $\mathbf{I}$ is the unit matrix.

The cubic symmetry implies that (up to the second order in the strain components) the free energy change corresponding to a thermodynamically reversible elastic deformation under an external pressure $p$ reads [2]

$$
\begin{gathered}
\Delta F / V_{p}=-p\left(\varepsilon_{x x}+\varepsilon_{y y}+\varepsilon_{z z}\right)+ \\
+\frac{1}{2} C_{11}\left(\varepsilon_{x x}^{2}+\varepsilon_{y y}^{2}+\varepsilon_{z z}^{2}\right)+ \\
+C_{12}\left(\varepsilon_{x x} \varepsilon_{y y}+\varepsilon_{y y} \varepsilon_{z z}+\varepsilon_{x x} \varepsilon_{z z}\right)+ \\
+2 C_{44}\left(\varepsilon_{x y}^{2}+\varepsilon_{y z}^{2}+\varepsilon_{x z}^{2}\right)
\end{gathered}
$$

where $V_{p}$ is the reference (equilibrium) volume of the system at the pressure $p$, and $\mathrm{C}_{11}, \mathrm{C}_{12}, C_{44}$ are the components of the tensor of elastic constants in the Voigt notation.

In the case of the f.c.c. phase it is convenient to consider deformations of cubic samples of $N=4 n^{3}$ particles because they exhibit the symmetry of the phase. It is also convenient to choose the box edges parallel to the unit cell and to take the reference box of the form $H_{x x}=H_{y y}=H_{z z}=L_{p}, H_{x y}=H_{y z}=H_{x z}=0$, where $L_{p}=V_{p}^{1 / 3}$ denotes the reference box edge.

It follows from (1) and (3) that three deformations are sufficient to determine all the elastic constants of the f.c.c. hard sphere crystal, e.g. one can use the following box matrices:

$$
\begin{aligned}
& \text { 1) } h_{x x}=h_{y y}=h_{z z}=\left(1+\delta_{1}\right) L_{p}, \\
& \text { 2) } h_{x x}=\left(1+\delta_{2}\right) L_{p}, h_{y y}=\left(1-\delta_{2}\right) L_{p}, \\
& \text { 3) } h_{x y}=\delta_{3} L_{p}
\end{aligned}
$$

where $\delta_{i}$ are small reals, and the matrix elements which remain unchanged with respect to the reference state are not specified. Elementary calculations show that:

$$
\begin{gathered}
\frac{1}{V_{p}} \frac{\partial F}{\partial \delta_{1}} \mid \delta_{1}=0=-3 p \\
\frac{1}{V_{p}} \frac{\partial^{2} F}{\partial \delta_{1}^{2} \mid \delta_{1}=0}=3\left(C_{11}+2 C_{12}-p\right)
\end{gathered}
$$




$$
\begin{gathered}
\frac{1}{V_{p}} \frac{\partial^{2} F}{\partial \delta_{2}{ }^{2}} \mid \delta_{2}=0=2\left(C_{11}-C_{12}-p\right), \\
\frac{1}{V_{p}} \frac{\partial^{2} F}{\partial \delta_{3}{ }^{2} \delta_{3}=0}=2\left(2 C_{44}-p\right),
\end{gathered}
$$

what is sufficient to obtain $p, \mathrm{C}_{11}, \mathrm{C}_{12}, C_{44}$.

The free energy of the system in the perfect and deformed cubic box can be calculated by the Frenkel-Ladd method [26]. The obtained results can be then numerically differentiated to determine the pressure and the elastic constants.

\section{NpT SIMULATIONS IN A PERIODIC BOX OF VARIABLE SHAPE}

The NpT simulations discussed below follow the Parrinello-Rahman idea of averaging strain fluctuations [8]. That idea has been further developed in the references [10, 11], The version of this method applied here is based on [20] where the general formalism was sketched. Below the method is illustrated in more detail for the case of the cubic solid.

At constant pressure, $p$, it is convenient to expand the free enthalpy (Gibbs free energy) [31] as no linear terms in the strain components are present in this expansion

$$
\begin{gathered}
\Delta G / V_{p}=\Delta\left(F_{\text {elastic }}+p V\right) / V_{p}= \\
=\frac{1}{2} B_{11}\left(\varepsilon_{x x}^{2}+\varepsilon_{y y}^{2}+\varepsilon_{z z}^{2}\right)+ \\
+B_{12}\left(\varepsilon_{x x} \varepsilon_{y y}+\varepsilon_{y y} \varepsilon_{z z}+\varepsilon_{x x} \varepsilon_{z z}\right)+ \\
+2 B_{44}\left(\varepsilon_{x y}^{2}+\varepsilon_{y z}^{2}+\varepsilon_{x z}^{2}\right),
\end{gathered}
$$

where $V$ is the volume of the deformed system, the reference state corresponds to the equilibrium state at $p$, and the elastic constants $B_{i j}$ and $C_{i j}[2,31]$ fulfill the relations:

$$
B_{11}=C_{11}-p, \quad B_{12}=C_{12}+p, \quad B_{44}=C_{44}-p .
$$

It follows from [8] that

$$
\begin{aligned}
\left\langle\varepsilon_{q \alpha \alpha}^{2}\right\rangle & =\frac{k T}{V_{p}} \frac{B_{11}+B_{12}}{B_{11}^{2}+B_{11} B_{12}-2 B_{12}^{2}}, \\
\left\langle\varepsilon_{\alpha \alpha} \varepsilon_{\beta \beta}\right\rangle & =-\frac{k T}{V_{p}} \frac{B_{12}}{B_{11}^{2}+B_{11} B_{12}-2 B_{12}^{2}},
\end{aligned}
$$




$$
\left\langle\varepsilon_{\alpha \beta}^{2}\right\rangle=-\frac{k T}{V_{p}} \frac{1}{4 B_{44}}
$$

where $\alpha, \beta=x, y, z, \alpha \neq \beta$, and

$$
\langle f\rangle=\frac{\int d \varepsilon^{(6)} f \exp \left(-\frac{G}{k T}\right)}{\int d \varepsilon^{(6)} \exp \left(-\frac{G}{k T}\right)} .
$$

The integration in the formula (13), which concerns all the independent components of the strain tensor

$$
d \varepsilon^{(6)} \quad=\quad d \varepsilon_{x x} d \varepsilon_{y y} d \varepsilon_{z z} d \varepsilon_{x y} d \varepsilon_{y z} d \varepsilon_{x y}
$$

can be converted to integration over the independent elements of the box matrix

$$
d \mathbf{h}^{(6)} \quad=\quad d h_{x x} d h_{y y} d h_{z z} d h_{x y} d h_{y z} d h_{x z},
$$

what gives

$$
\langle f\rangle=\frac{\int d \mathbf{h}^{(6)} j(\mathbf{h}, \mathbf{H}) f \exp \left(-\frac{G}{k T}\right)}{\int d \mathbf{h}^{(6)} j(\mathbf{h}, \mathbf{H}) \exp \left(-\frac{G}{k T}\right)},
$$

where the Jacobian

$$
j(\mathbf{h}, \mathbf{H}) \equiv \frac{\partial\left(\dot{\varepsilon}_{x x}, \varepsilon_{y y}, \varepsilon_{z z}, \varepsilon_{x y}, \varepsilon_{y z}, \varepsilon_{x z}\right)}{\partial\left(h_{x x}, h_{y y}, h_{z z}, h_{x y}, h_{y z}, h_{x z}\right)}
$$

of the transformation $\mathbf{h} \rightarrow \mathrm{e}$ has the form [32]

$$
j(\mathbf{h}, \mathbf{H})=j_{1}(\mathbf{h}) j_{2}(\mathbf{H}),
$$

and

$$
\begin{gathered}
j_{1}(\mathbf{h})=\operatorname{det}(\mathbf{h})\left[\operatorname{Tr}\left(\begin{array}{lll}
\mathbf{h} & \mathbf{h} & \mathbf{h}
\end{array}\right)-\operatorname{Tr}(\mathbf{h})^{3}\right], \\
j_{2}(\mathbf{h})=\operatorname{det}(\mathbf{H})^{-4} / 8
\end{gathered}
$$

It is worth to notice that also for asymmetric box matrices of the form discussed in [14] the Ja-cobian separates into factors which depend only on $\mathbf{h}, \mathbf{H}$, respectively.

It follows from (15) that (14) can be rewritten in the form

$$
\langle f\rangle=\frac{\int d \mathbf{h}^{(6)} j_{1}(\mathbf{h}) f \exp \left(-\frac{G}{k T}\right)}{\int d \mathbf{h}^{(6)} j_{1}(\mathbf{h}) \exp \left(-\frac{G}{k T}\right)} .
$$


what means that the simulations can be performed without knowing the reference state in advance [32],

One may add that the bulk modulus

$$
B \equiv-V \frac{\partial p}{\partial V}=\frac{B_{11}+2 B_{12}}{3}
$$

can be obtained by averaging the volume fluctuations

$$
\left\langle\left(V-V_{p}\right)^{2}\right\rangle=\frac{k T V_{p}}{B} .
$$

\section{SUMMARY AND CONCLUSIONS}

The advantage of the two methods described above with respect to other methods is that the former do not require evaluation of the microscopic expressions for the pressure (or stress tensor) or the elastic constants. This can be an important advantage if such expressions are non-trivial to obtain and to apply in simulations. It is so indeed in certain cases, e.g. for some complex molecules or for molecules which interact through certain non-analytic potentials. In such cases, simplicity of the approaches described, especially in the case of the NpT simulations, encourages one to use them for determining the elastic properties of interest. Various tests are, however, necessary to check if, and to what extent, these methods can be useful in practical simulations. Results of one of such tests are presented in the following article [33], where elastic properties of the hard sphere system are considered. Other tests will be described in future publications.

\section{Acknowledgments}

Part of this work was supported by the grant 4T11F01023 of the Polish Committee for Scientific Research $(\mathrm{KBN})$.

\section{References}

[1] M. Born, K. Huang, Dynamical Theoiy of Ciystal Lattices, Oxford University Press, Oxford, 1954.

[2] D. C. Wallace. Thermodynamics of Crystals, Wiley, New York, 1972.

[3] J. H. Weiner, Statistical Mechanics of Elasticity (Wiley, New York. 1983).

[4] L. D. Landau, E. M. Lifshits, A. M. Kosevich, I. P. Pitaevskii, Theoiy of Elasticity, Pergamon Press, London, 1986.

[5] D. R. Squire, A. C. Holt, W. G. Hoover, Physica (Amsterdam) 42, 388 (1968).

[6] W. G. Hoover, Z. W. Salsburg, J. Chem. Phys. 54, 2129 (1971).

[7] J. Q. Broughton, G. H. Gilmer, J. D. Weeks, Phys. Rev. B25, 4651 (1982).

[8] M. Parrinello, A. Rahman, J. Chem. Phys. 76, 2662 (1982).

[9] M. Sprik, R. W. Impey, M. L. Klein, Phys. Rev., B29, 4368 (1984).

[10] J. R. Ray, A. Rahman, J. Chem. Phys., 80, 4423 (1984). 
[11] J. R. Ray, A. Rahman, J. Chem. Phys., 82, 4243 (1985).

[12] J. R.. Ray, M. C. Moody, A. Rahman, Phys. Rev., B32, 733 (1985).

[13] J. R. Ray, M. C. Moody, A. Rahman,- Phys. Rev., B33, 895 (1986).

[14] K. W. Wojciechowski, Molec, Phys., 61, 1247 (1987).

[15] D. Frenkel, A. J. C. Ladd, Phys. Rev! Lett., 59, 1169 (1987).

[16] K. J. Runge, G. V. Chester, Phys. Rev. A36, 4852 (1987).

[17] T. Çagin, J. R. Ray, Phys. Rev., B38, 7940 (1988).

[18] K. W. Wojciechowski, A. C. Branka, Phys. Lett., A134, 314 (1989).

[19] D. H. Boal, U. Seifert, J. C. Shillcock, Phys. Rev., E48, 4274 (1993).

[20] K. W. Wojciechowski, K. V. Tretiakov, Comput. Phys. Commun,. 121-122, 528 (1999).

[21] O. Farago, Y. Kantor, Phys. Rev., E61, 2478 (2000).

[22] S. Sengupta, P. Nielaba, M. Rao, K. Binder, Phys. Rev. E61, 1072 (2000).

[23] M. A. Bates, D. Frenkel, Phys. Rev., E61, 5223 (2000).

[24] Z. Zhou, J. Chem. Phys., 114, 8769 (2001).

[25] M. Bowick, A. Cacciuto, G. Thorleifsson, A. Travesset, Phys. Rev. Lett., 87, 148103 (2001).

[26] D. Frenkel, A. J. C. Ladd, J. Chem. Phys., 18, 3188 (1984).

[27] K. V. Tretiakov, K. W. Wojciechowski, Phys. Rev., E60, 7626 (1999).

[28] K. V. Tretiakov, K. W. Wojciechowski, J. Phys.: Cond. Matter, 14, 1261 (2002).

[29] K. W. Wojciechowski, K. V. Tretiakov, M. Kowalik, to be published.

[30] K. W. Wojciechowski, K. V. Tretiakov, A. C. Brańka, M. Kowalik, to be published.

[31] K. W. Wojciechowski, Mol. Phys. Rep., 10, 129 (1995).

[32] K. W. Wojciechowski, Simple and efficient method of elastic properties determination by computer simulation, in Proc. IX Workshop of the Polish Soc. Computer Simul., Koszalin 28-31.08.2002, in print (in Polish).

[33] K. W. Wojciechowski, K. V. Tretiakov, Comput. Meth. Sci. Technol., 8(2), 84-92 (2002). 